\title{
PARESTESIA E/OU DOR NAS MÃOS E/OU PUNHOS COMO MOTIVO DE ENCAMINHAMENTO PARA ESTUDO ELETRONEUROMIOGRÁFICO
}

\author{
Carmelinda Correia de Campos ${ }^{1}$, Gilberto Mastrocola Manzano², \\ Adauto Castelo Filho ${ }^{3}$, João Antonio Maciel Nóbrega²
}

\begin{abstract}
RESUMO - Com o objetivo de conhecermos os motivos de encaminhamento para estudo eletrofisiológico, perguntamos a 490 pacientes, encaminhados a dois laboratórios de neurofisiologia (UNIFESP e UNEF): "Qual o motivo que a(o) levou a procurar o médico e que fez com que ele solicitasse este exame"? Parestesia e/ou dor nas mãos e/ou punhos foram espontaneamente referidas como motivo principal por 175 deles (26\% UNIFESP e 40\% UNEF). O exame eletroneuromiográfico foi normal em, respectivamente, 30,8\% e 35,3\% e revelou compressão do nervo mediano no carpo em $59 \%$ e $51,5 \%$ destes pacientes. Sintomas noturnos e parestesia foram comuns nos dois grupos de pacientes, mas não foram capazes de discriminar aqueles com e sem compressão do nervo mediano no carpo. O sintoma dor predominou nos pacientes com exame normal. A pergunta formulada pareceu ser bom instrumento de seleção. Conseguiu selecionar duas amostras de pacientes sintomáticos semelhantes, em laboratórios com população alvo muito diferente.
\end{abstract}

PALAVRAS-CHAVE: parestesia e dor nas mãos, síndrome do túnel do carpo, compressão do nervo mediano no carpo, condução nervosa.

\begin{abstract}
Paresthesia and/or pain affecting the hand and/or wrist as a referral for electrodiagnostic studies
ABSTRACT - To study the reasons for referral to electrodiagnostic evaluation, 490 patients referred for electrodiagnostic evaluation to two laboratories (UNIFESP and UNEF) answered the following question: "What was the reason that brought you to look for a physician and that led him to ask this examination?" Paresthesia and/or pain were answered as the main reason by 175 of them ( $26 \%$ UNIFESP and $40 \%$ UNEF). The electromyographical examinations were normal in $30.8 \%$ and $35.3 \%$ and involvement of the median nerve at the wrist was detected in $59 \%$ and $51.5 \%$ of them. Nocturnal symptoms and paresthesia were commonly noted in both groups of patients; they, however, were not able to separate the patients with and without median nerve compression at the wrist. The question asked seems to be a useful selection instrument, to select two similar symptomatic samples of patients from two laboratories serving with very different target populations.
\end{abstract}

KEY WORDS: paresthesia and pain in hands, carpal tunnel syndrome, compression of the median nerve at the wrist, nerve conduction.

Parestesia e/ou dor nas mãos e/ou punhos são queixas comuns entre pacientes encaminhados para exame eletrofisiológico e com frequência fazem parte de um conjunto de sinais e sintomas, conhecido com síndrome do túnel do carpo (STC) ${ }^{1}$.

Desde que Brain et al. (1947) $)^{2}$ e Phalen (1951) ${ }^{3}$ descreveram os principais sintomas da compressão do nervo mediano no carpo (CNMC), e desde que a esse conjunto de sintomas foi dado o nome de síndrome do túnel do carpo ${ }^{4}$, esta síndrome passou a ser usada na identificação clínica de pacientes com
CNMC. Em algumas publicações, a STC é utilizada como sinônimo de tal compressão $0^{5,6}$. No entanto, outras afecções, tais como radiculopatias, plexopatias, polineuropatias, lesões ósteo-articulares, lesões corticais, e compressões do próprio nervo mediano em outros locais são citadas na literatura como possíveis causadoras dos mesmos sintomas ${ }^{7-9}$, devendo ser consideradas no diagnóstico diferencial ${ }^{10}$. Isso torna o diagnóstico de CNMC, baseado apenas em sinais e sintomas clínicos, não totalmente confiável, sendo o exame eletrofisiológico considerado instru-

Estudo realizado no Setor de Neurofisiologia Clínica da Disciplina de Neurologia da Universidade Federal de São Paulo - Escola Paulista de Medicina (UNIFESP-EPM), São Paulo SP, Brasil: 'Pós Graduanda da Disciplina de Neurologia; ${ }^{2}$ Professor Adjunto da Disciplina de Neurologia; ${ }^{3}$ Professor Adjunto da Disciplina de Epidemiologia Clínica.

Recebido 16 Abril 2002, recebido na forma final 20 Julho 2002. Aceito 10 Agosto 2002. 
mento de extrema importância na sua confirmação e no diagnóstico diferencial ${ }^{11-13}$.

O objetivo do presente estudo foi: a) avaliar a ocorrência de parestesia e/ou dor nas mãos e/ou punhos como causa primária de encaminhamento para exame eletroneuromiográfico, segundo a informação do paciente, em dois laboratórios de neurofisiologia: Universidade Federal de São Paulo - Escola Paulista de Medicina (UNIFESP-EPM), hospital universitário, e Unidade de Diagnósticos Eletrofisiológicos (UNEF), clínica em região industrial; b) determinar a prevalência de CNMC em pacientes com queixas de parestesia e/ou dor nas mãos e/ou punhos.

\section{MÉTODO}

A pesquisa foi aprovada pelo Comitê de Ética em Pesquisa da Universidade Federal de São Paulo (UNIFESP), protocolo $n^{\circ} 721 / 99$.

Fase a: durante quatro meses, fizemos a 150 pacientes da UNIFESP e 340 da UNEF a seguinte pergunta: "Qual o motivo que o levou a procurar o médico e que fez com que ele solicitasse este exame?"

Fase $b$ : todos os pacientes foram submetidos a avaliação eletrofisiológica rotineira, utilizando as técnicas convencionais ${ }^{6}$ necessárias para o diagnóstico. Porém, naqueles em que a resposta espontânea à pergunta citada incluiu queixas de parestesia e/ou dor envolvendo mãos e/ou punhos (excluindo apenas os pacientes com traumatismos e cirurgias anteriores), aplicamos o seguinte protocolo: a) estudo da condução sensitiva: no nervo mediano ( $2^{\circ}$ e $3^{\circ}$ dedos e palma-punho), no nervo ulnar $\left(5^{\circ}\right.$ dedo) e nervo radial $\left(1^{\circ}\right.$ dedo); b) estudo da condução motora: no nervo mediano e ulnar, e no nervo radial sempre que considerado necessário; c) testes considerados mais sensíveis, como comparação das latências dos picos negativos dos potenciais sensitivos, obtidos com a estimulação do nervo mediano e ulnar nos seus segmentos palma - punho $(8 \mathrm{~cm})^{8}$, mediano e radial nos segmentos punho - $1^{\circ}$ dedo $(10 \mathrm{~cm})$ e mediano e ulnar nos segmen- tos punho $-4^{\circ}$ dedo $(13 \mathrm{~cm})$ seguindo a metodologia descrita por Johnson ${ }^{14,15}$. Estes testes foram realizados em pacientes com suspeita de CNMC, sempre que os chamados testes convencionais resultaram normais.

O estudo da condução sensitiva foi antidrômico, exceto nos segmentos palma-punho, e a temperatura das mãos foi mantida igual a $32^{\circ} \mathrm{C}$ ou acima.

Neste grupo, a ocorrência de queixas de dor, parestesia e presença de sintomas noturnos, envolvendo as mãos e/ou punhos foi avaliada e sua correlação com a presença ou não de CNMC foi calculada utilizando-se o teste do Qui-quadrado.

\section{RESULTADOS}

As respostas à pergunta apresentada permitiram classificar os motivos de encaminhamento de acordo com os segmentos corporais envolvidos; motivos decorrentes de cirurgias ou traumas prévios foram separados em grupo independente (Tabela 1).

Após esta abordagem global, foram analisados em detalhe os resultados dos pacientes cujas queixas espontâneas incluíam mãos e/ou punhos. A incidência destes pacientes correspondeu a $26 \%$ dos da UNIFESP e $40 \%$ dos da UNEF e os resultados da avaliação eletroneuromiográfica estão demostrados na Tabela 2.

A Tabela 3 amostra a síntese dos resultados da avaliação eletrofisiológica nos pacientes provenientes dos dois laboratórios, com ênfase nos exames normais, naqueles compatíveis com CNMC e outros diagnósticos.

As Tabelas 4, 5 e 6 mostram a incidência de dor, parestesia e sintomas noturnos nos pacientes com CNMC e com exames normais. A correlação destes sintomas com a presença ou não de CNMC foi obtida pelo teste do Qui-quadrado. Observamos que dor, como sintoma isolado, foi significantemente menos frequente nos pacientes com CNMC $\left(\chi^{2}\right.$ gl1 $=5,26$ $\mathrm{p}=0,022)$. Parestesia, como sintoma isolado $\left(\chi^{2} \mathrm{gl} 1=0,13\right.$ $p=0,721)$, e sintomas noturnos ( $\chi^{2}$ gl $1=1,49 p=0,222$ ) não foram significantemente diferentes nos dois grupos.

Tabela 1. Resultados percentuais dos motivos de encaminhamento para estudo eletroneuromiográfico, obtidos segundo informações dos 490 pacientes.

\begin{tabular}{lcc}
\hline Motivo do encaminhamento & UNIFESP & UNEF \\
\hline Sintomas acometendo MMSS & $42 \%$ & $57 \%$ \\
Sintomas acometendo coluna e MMII & $25 \%$ & $27 \%$ \\
Sintomas acometendo MMSS e MMII & $18 \%$ & $3 \%$ \\
Sintomas acometendo face, visão, audição e outros & $13 \%$ & $10 \%$ \\
Cirurgias anteriores e traumas & $2 \%$ & $3 \%$ \\
\hline
\end{tabular}

MMSS, membros superiores; MMII, membros inferiores; UNIFESP, Universidade Federal de São Paulo; UNEF, Unidade de Diagnósticos Eletrofisiológicos. 
Tabela 2. Resultados dos exames eletroneuromiográficos dos 175 pacientes com queixas envolvendo mãos e/ou punhos.

\begin{tabular}{lcc}
\hline ENMG & UNIFESP & UNEF \\
\hline CNMC & 23 & 70 \\
CNMC + polineuropatia diabética & 1 & 3 \\
CNMC + radiculopatia & 0 & 4 \\
CNMC + mononeuropatia múltipla & 0 & 2 \\
CNMC + lesão do nervo ulnar & 0 & 1 \\
Normais & 12 & 48 \\
Radiculopatias & 2 & 6 \\
Lesão do nervo ulnar & 1 & 1 \\
Plexopatias & 0 & 1 \\
\hline
\end{tabular}

CNMC, compressão do nervo mediano no carpo; ENMG, eletroneuromiografia; UNIFESP, Universidade Federal de São Paulo; UNEF, Unidade de Diagnósticos Eletrofisiológicos.

Tabela 3. Distribuição percentual, dos resultados dos exames eletroneuromiográficos dos 175 pacientes selecionados nos dois serviços com queixas envolvendo mãos e/ou punhos.

\begin{tabular}{lcccc}
\hline & $\mathrm{N}$ & CNMC & Normais & Outros \\
\hline UNIFESP & 39 & $59,0 \%$ & $30,8 \%$ & $10,2 \%$ \\
UNEF & 136 & $51,5 \%$ & $35,3 \%$ & $13,2 \%$ \\
\hline
\end{tabular}

CNMC, compressão do nervo mediano no carpo; N, número de pacientes; outros, outros diagnósticos . UNIFESP,Universidade Federal de São Paulo; UNEF, Unidade de Diagnósticos Eletrofisiológicos.

Tabela 4. Incidência da dor, como sintoma isolado, em pacientes com compressão do nervo mediano no carpo e com exames normais.

\begin{tabular}{lccc}
\hline $\begin{array}{l}\text { Sintomas } \\
\text { (mãos e punhos) }\end{array}$ & C/CNMC & Normais & Total \\
\hline Dor & 5 & 10 & 15 \\
Outros & 88 & 50 & 138 \\
Total & 93 & 60 & 153 \\
\hline
\end{tabular}

C/CNMC, com compressão do nervo mediano no carpo; outros = outros sintomas. $x^{2} g \mid 1=5,26 ; p, 0,022$.

Tabela 5. Incidência das parestesias, como sintoma isolado, em pacientes com compressão do nervo mediano no carpo e com exames normais.

\begin{tabular}{lccc}
\hline $\begin{array}{l}\text { Sintomas } \\
\text { (mãos e punhos) }\end{array}$ & C/CNMC & Normais & Total \\
\hline Parestesias & 33 & 23 & 56 \\
Outros & 60 & 37 & 97 \\
Total & 93 & 60 & 153 \\
\hline
\end{tabular}

C/CNMC, com compressão do nervo mediano no carpo; outros, outros sintomas. $x^{2}$ gl1 $=0,13 p, 0,721$
Tabela 6. Incidência de sintomas noturnos em pacientes com compressão do nervo mediano no carpo e com exames normais.

\begin{tabular}{lccc}
\hline $\begin{array}{l}\text { Sintomas } \\
\text { (mãos e punhos) }\end{array}$ & C/CNMC & Normais & Total \\
\hline Sintomas noturnos & 45 & 23 & 68 \\
Outros & 48 & 37 & 85 \\
Total & 93 & 60 & 153 \\
\hline
\end{tabular}

C/CNMC, com compressão do nervo mediano no carpo; outros, outros sintomas. $x^{2} g \mid 1=1,49 p, 0,222$.

\section{DISCUSSÃO}

A pergunta quanto ao motivo que levou à indicação do exame eletroneuromiográfico, da maneira como foi formulada, mostrou-se capaz de selecionar grupos de pacientes cujos resultados, no que se refere às causas determinantes desses sintomas, foram semelhantes nos dois serviços. Isto pode ser observado nos resultados dos exames eletrofisiológicos citados na Tabela 3. Por esses resultados, verificamos que a maioria dos pacientes, com queixas de parestesia e/ou dor nas mãos e/ou punhos, era portadora de CNMC $(59,0 \%$ na UNIFESP e $51,5 \%$ na UNEF), como já era esperado. Polineuropatias, radiculopatias, plexopatias e lesões de outros nervos causaram os mesmos sintomas num pequeno número de pacientes $(10,2 \%$ na UNIFESP e $13,2 \%$ na UNEF), como mencionado na literatura ${ }^{7-10}$. Surpreendeu-nos o grande número de pacientes sintomáticos nos quais os exames eletrofisiológicos foram normais (30,8\% na UNIFESP e 35,3\% na UNEF).

Os estudos publicados, abordando pacientes com queixas nos membros superiores, ou não mencionam como os pacientes foram selecionados ou, se o fazem, não informam os resultados dos exames eletrofisiológicos, exceto quanto ao número de pacientes em que a CNMC foi confirmada ou excluída ${ }^{11,12,16 .}$

Katz et al. ${ }^{17}$ examinaram pacientes com queixas nos membros superiores e encontraram incidências diferentes das nossas, principalmente no que se refere ao número de pacientes com outros diagnósticos $(40 \%$ de CNMC, $26,4 \%$ de normais e $37 \%$ com outros diagnósticos). Essas diferenças devem-se, provavelmente, aos critérios utilizados na seleção dos pacientes. Nós, mediante uma pergunta, identificamos a população de interesse como sendo aquela com queixa principal de parestesia e/ou dor nas mãos e/ou punhos. Já esses autores não mencionaram os critérios utilizados, referindo apenas que eram pacientes com queixas nos membros superiores. 
Em outro estudo, Katz et al..$^{12}$ examinaram pacientes que preencheram os critérios clínicos da STC relacionada ao trabalho, estipulados pelo National Institute for Occupational Safety and Health $\mathrm{NIOSHI}$. Eles referem que a metade do número de pacientes portador de sinais e sintomas da STC não tinha alterações eletrofisiológicas compatíveis com CNMC. Porém, não dão detalhes quanto aos resultados dos exames eletrofisiológicos desses pacientes. O mesmo pode ser observado na publicação feita por Boniface et al. ${ }^{16}$, que examinaram 100 pacientes com diagnóstico clínico de STC e encontraram alterações eletrofisiológicas confirmando o diagnóstico de CNMC em 64 deles. Não é possível, pelos dados fornecidos, saber a ocorrência de exames normais ou de outras afecções nos demais pacientes.

Nos nossos resultados, observamos que parestesia e sintomas noturnos, considerados sintomas primários da STC por Nathan et al. ${ }^{18}$ e You et al. ${ }^{6}$, e que são rotineiramente atribuídos à compressão neural, estavam presentes em número expressivo de pacientes com exames eletrofisiológicos normais, não mostrando correlação significante com a CNMC (Tabelas 5 e 6). Já o sintoma dor (Tabela 4), considerado sintoma secundário da STC 6,18 , predominou nos pacientes sintomáticos com exames normais.

A dor como sintoma isolado foi menos frequente nos pacientes com CNMC, acometendo $5,4 \%$ deles. Essa Incidência foi maior que a de 1,5\% encontrada por Kouyoumdjian ${ }^{19}$.

As causas desses sintomas, em pacientes cujos exames foram normais, não estão esclarecidas. A possibilidade de haver, entre eles alguns nos quais os exames eletrofisiológicos tenham sido falso-negativos deve ser considerada ${ }^{20}$. Porém, esse número deve ser pequeno, já que utilizamos testes eletrofisiológicos tidos como mais sensíveis ${ }^{21-25}$. Questionamo-nos se estes sintomas poderiam estar associados a alterações osteoarticulares, já que a maioria desses pacientes trabalha na indústria, onde a incidência dessas alterações, assim como a CNMC, é considerada maior que na população geral ${ }^{26}$, embora esta associação continue controversa ${ }^{27-29}$.

Conclusão - Parestesia e/ou dor nas mãos e/ou punhos foram motivos frequentes de encaminhamento para exame eletrofisiológico, na nossa amostra. A avaliação eletrofisiológica revelou que CNMC foi a principal causa desses sintomas, mas número significativo de pacientes mostrou ter exames normais.
A incidência de sintomas noturnos, parestesia e dor, nesta amostra de pacientes surpreendeu-nos, e merece futuras pesquisas, uma vez que os chamados sintomas primários da STC não mostraram associação significante com CNMC, enquanto o sintoma dor predominou no grupo de pacientes sintomáticos com exames normais.

A pergunta utilizada mostrou ser capaz de identificar um grupo homogêneo de pacientes sintomáticos, com a mesma distribuição causal, nos dois serviços com população alvo bastante diferente, podendo ser útil na padronização de futuras pesquisas e ser utilizada como instrumento epidemiológico de seleção, bastando para isso ser testada quanto à sua reprodutibilidade.

\section{REFERÊNCIAS}

1. Stevens JC, Smith BE, Weaver AL, Bosch EP, Deen HG, Wilkens J A Symptoms of 100 patients with electromyographically verified carpal tunnel syndrome. Muscle Nerve 1999; 22:1448-1456.

2. Brain WR, Wright AD, Wilkinson M. Spontaneous compression of both median nerves in the carpal tunnel: six cases treated surgically. Lancet 1947;1:277-282.

3. Phalen GS. Spontaneous compression of the median nerve at the wrist. JAMA 1951;145:1128-1132.

4. Phalen GS. The carpal tunnel syndrome: seventeen years' experience in diagnosis and treatment of six hundred fifty-four hands. J Bone Joint Surg 1966; 48A:211-228.

5. Dawson DM, Hallett M. Entrapment neuropathies. 3.Ed. Philadelphia: Lippincott Raven, 1999:20-94.

6. Kimura J. Electrodiagnosis in diseases of nerve and muscle. 2.Ed. Philadelphia: F.A.Davis 1989:501-504.

7. American Association of Electrodiagnostic Medicine, American Academy of Neurology, American Academy of Physical Medicine Rehabilitation. Literature review of the usefulness of nerve conduction studies and electromyography for evaluation of patients with carpal tunnel syndrome. Muscle Nerve 1993;16:1392-1414.

8. Stevens JC. AAEE minimonograph: the electrodiagnosis of carpal tunnel syndrome. Muscle Nerve 1997;20:1477-1486.

9. You H, Simmons Z, Freivalds A, Kothari MJ, Naidu SH. Relationships between clinical symptom severity scales and nerve conduction measures in carpal tunnel syndrome. Muscle Nerve 1999;22:497-501.

10. Quality Standards Subcommittee of the American Academy of Neurology. Practice parameter for carpal tunnel syndrome. Neurology 1993;43:2406-2409.

11. Gerr F, Letz R.The sensitivity and specificity of tests for carpal tunnel syndrome vary with the comparison subjects. J Hand Surg 1998; 23B:151-155.

12. Katz JN, Larson MG, Fossel AH, Liang MH. Validation of surveillance case definition of carpal tunnel syndrome. Am J Public Health 1991;81: 189-192.

13. Wilcox MS, Bilbao A. Sensitivity of electrophysiological studies and the carpal tunnel syndrome. Muscle Nerve 1993;67:1265-1266.

14. Johnson EW, Kukla RD, Wongsan PE, Piedmonta A. Sensory latencies to the ring finger: normal values and relation to carpal tunnel syndrome. Arch Phys Med Rehabil 1981;62: 206-208.

15. Johnson EW, Sipski M, Lamimrtse T. Median and radial sensory latencies to digit I: normal values and usefulness in carpal tunnel syndrome. Arch Phys Med Rehabil 1987;68:140-141.

16. Boniface SJ, Morris I. MacLeod A. How does neurophysiological assessment influence the management and outcome of patients with carpal tunnel syndrome? Br J Rheumat 1994;33:1169-1170.

17. Katz JN, Larson MG, Sabra A, et al. The carpal tunnel syndrome: diagnostic utility of the history and physical examination findings. Ann Intern Med 1990;112:321-327. 
18. Nathan PA, Meadows KD, Doyle SL. Relationship of age and sex to sensory conduction of the median nerve at the carpal tunnel and association of slowed conduction with symptoms. Muscle Nerve 1988;11:1149-1153.

19. Kouyoumdjian JA. Síndrome do túnel do carpo, aspectos clínicoepidemiológicos em 668 casos. Arq Neuropsiquiatr 1999;57:202-207.

20. Rempel DM, Harrison RJ, Barnhartn S. Work-related cumulative trauma disorders of the upper extremity. JAMA 1992;267:6:838-842.

21. Uncini A, Di Muzio A, Awad DJ, Manente G, Tafuro M, Gambi D. Sensitivity of three median-to-ulnar comparative tests in diagnosis of mild carpal tunnel syndrome. Muscle Nerve 1993;16:366-1373.

22. Buchthal F, Rosenfalck A, Trojaborg W. Electrophysiological findings in entrapment of the median nerve at wrist and elbow. J Neurol Neurosurg Psychiatry 1974;37: 340-360.

23. Jackson DA, Cliffor JC. Electrodiagnosis of mild carpal tunnel syndrome. Arch Phys Med Rehabil 1989;70:199-204.
24. Pease WS, Cannel CD, Johnson EW. Median to radial latency difference test in mild carpal tunnel syndrome. Muscle Nerve 1989;12:905-909.

25. Uncini A, Lange DJ, Solomon M, Soliven B, Meer J, Lovelace RE. Ring finger testing in carpal tunnel syndrome: a comparative study of utility. Muscle Nerve 1989;12:735-741.

26. Barnhart S, Demers PA, Miller M, Longstreth WT, Rosenstock L. Carpal tunnel syndrome among ski manufacturing workers. Scand J Work Envirom Health 1991;17:46-52.

27. Hadler NM, Chapel H. Repetitive upper-extremity motions in the workplace are not hazardous. J Hand Surg 1997;22A:19-29.

28. Vender MI, Kasdan ML, Truppa K. Upper extremity disorders: A literature review to determine work relatedness. J. Hand Surg 1995; 20A :534-541.

29. Nathan PA, Meadows KD, Doyle SL. Occupation as a risk factor for impaired sensory conduction of the median nerve at the carpal tunnel. J Hand Surg 1988;13B:167-170. 